\title{
Spatial Patterns of SSSEP Under the Selective Attention to Tactile Stimuli in Each Hand
}

\author{
Yunjun Nam*, Bonkon Koo*, and Seungjin Choi ${ }^{* \dagger}$ \\ ${ }^{*}$ School of Interdisciplinary Bioscience and Bioengineering \\ ${ }^{\dagger}$ Department of Computer Science and Engineering \\ Pohang University of Science and Technology, Korea \\ \{druid, bkkoo, seungjin\}@ postech.ac.kr
}

\begin{abstract}
Steady-state somatosensory evoked potential (SSSEP) is a recently developing brain-computer interface (BCI) paradigm where the brain response to tactile stimulation of a specific frequency is used. Generally, SSSEP-based BCI classifies whether the subjects focus on the tactile stimulation at the fingers in the left or right hand. In this paper, we examine the spatial pattern difference in SSSEP from each kind of focuses. The experimental results showed that when the subjects focus to the stimulation at one hand, SSSEP response in the contralateral side decreases. These results could be related to the neurological background, that the sensory cortices for each hand is located in the contra lateral sides of the hands. We also investigate the spatial patterns obtained from common spatial pattern (CSP). In the survey results, the spatial filters, whose corresponding spatial pattern vectors emphasize the left and right parietal lobes, where the somatosensory cortices for each hand are located, made clearly separable clusters of the feature values for each class.
\end{abstract}

Keywords-electroencephalography (EEG); brain computer interface; steady-state somatosensory evoked potential (SSSEP); common spatial pattern

\section{INTRODUCTION}

The brain computer interface (BCI) is a system that provides a direct communication pathway between the brain and external devices by analyzing various brain signals [1]. Among various BCI techniques, steady-state somatosensory evoked potential (SSSEP)-based BCI has attracted the attention of BCI researchers, since its suitability as an interface was examined in [2]. SSSEP-based BCI analyzes the amplitude response of EEG signals for vibratory tactile stimuli. If a single tactile stimulation is given to a subject, an evoked potential with a specific waveform will be generated in the EEG signals. Likewise, if tactile stimulations are periodically applied in the form of a vibration with a specific frequency, the following evoked potentials will also be periodic. By examining this periodic response with time-frequency analysis, we can detect the type of the stimulation frequency being given to the subject. Müller-Putz et al. [2] reported that selective attention to a specific stimulus can modulate the induced SSSEP, and they exploited this paradigm to implement a novel BCI system.

They also mentioned about two unique advantages of SSSEP-based BCI against other BCI techniques. First, it observes the increase of power in the frequency bands of given tactile stimuli, so its signal response is easier to detect than the event-related desynchronization (ERD), which is observed as the power decrease of $\mu$-rhythm in the motor imagery BCI. Second, be compared to SSVEP-based BCI, SSSEP-based BCI is applicable even to the completely paralyzed patients, who lose the ability of voluntary gaze control. In many cases, the somatosensory system is more slowly affected than the motor system of persons suffering from most neuromuscular degenerative disorder.

To utilize above advantages, a series of researches was performed to implement and improve the SSSEP-based BCI. Dan Zhang et al. [3] developed a multi-modal BCI system, integrating the SSSEP and SSVEP-based BCIs, that can discriminate three kinds of mental tasks: attention to the 1) visual stimulus, 2) tactile stimulus at the right hand, and 3) the left hand. Ahn et al. [4] compared the performances of the motor imagery and SSSEP-based BCI, to investigate the feasibility of hybrid approach.

Recently, we [5] applied the common spatial pattern (CSP) method, which is one of the most intensively studied feature extraction method for the motor imagery BCI paradigm [6], [7], to SSSEP-based BCI. We pointed out that the distance between two somatosensory cortices corresponding to the left hand and right hand is sufficient to allow detection of the partial activations of each cortex. We assumed that the selective attention can evoke the partial activation of each cortex, and showed that CSP can extract the discriminative signal components emphasizing the spatial difference of the activations. We exploited the modified CSP approach, which applies CSP to the signals of each frequency band, to improve the performance of SSSEP-based BCI, and proved its usefulness.

However, we did not extensively considered about which channels (or brain regions) mainly contribute for the classification. We found the importance of C3 and C4 channels, but did not surveyed whether these channels actually show different responses to each attention or how much the differences are significant. In this paper, we examine the spatial pattern difference in SSSEP from each attention, to validate the usefulness of spatial information for SSSEP analysis and provide practical information for implementing SSSEP-based BCI system.

The rest of this paper is organized as follows. In Section II, we describe our experimental design for evoking and recording SSSEP, then explain the CSP method to analyze it. In Section III, we analyze the difference between the spatial patterns of SSSEP, recorded during the different attentions. We also 
present the discriminative spatial pattern of SSSEP obtained from CSP method, with its classification results. Finally, our results are summarized and discussed in Section IV.

\section{METHOD}

In this section, we describe the experimental design including the stimulation unit and EEG recording device. We also explain our signal processing method based on the CSP.

\section{A. Experimental Design}

To elicit SSSEP response, we used two round shapedvibration motors with a radius of $1 \mathrm{~cm}$ as the transducer. To control the transducers, $22 \mathrm{~Hz}$ and $27 \mathrm{~Hz}$ digital pulse signals were generated by a $\mathrm{C}++$ based program. $22 \mathrm{~Hz}$ signal was transmitted to the transducer attached on the thumb of the left hand, and $27 \mathrm{~Hz}$ signal was transmitted to the transducer attached on the thumb of the right hand. The EEG signals used in this study were recorded using a Biosemi Active Two ${ }^{\circledR}$ system. The sampling rate was $512 \mathrm{~Hz}$ and the signals in each trial were band-pass filtered between $0.5 \mathrm{~Hz}$ and $40 \mathrm{~Hz}$. With mentioned transducers and EEG recording device, we designed the BCI system, as graphically depicted in Fig. 1.

For each trial, two vibratory stimuli with different frequencies were administered to the subjects, having a duration of $10 \mathrm{~s}$. Before the stimuli were administered, one of the transducers vibrated for $2 \mathrm{~s}$ as the cue. The subjects were asked to concentrate on the transducer that was indicated by the cue. For every session, we collected 40 trials, half of which $(n=20)$ were assigned to the cue on the left hand, and half to the cue on the right hand. The set of trials assigned to the cue on the left and right hand are denoted below by $\boldsymbol{L}$ and $\boldsymbol{R}$, respectively. We examined the spatial pattern differences of SSSEP between $\boldsymbol{L}$ and $\boldsymbol{R}$ and tried the classification between them. We used two classification methods. As the basic approach, the first method used the amplitudes of raw signals as the classification feature. In the second method, we applied CSP to extract the discriminative signal components, then used their amplitudes as the features for the classification.

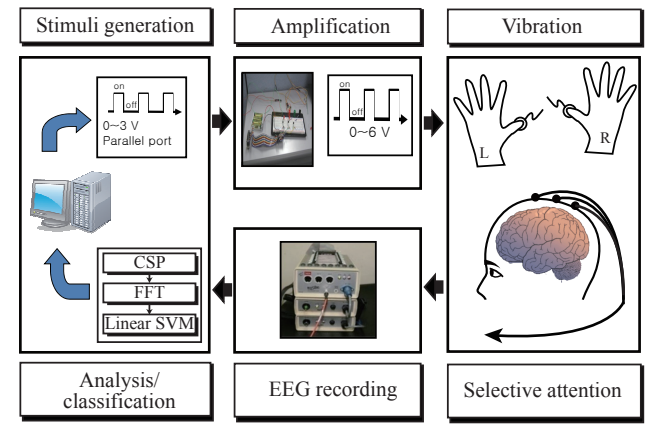

Fig. 1. Overview of the SSSEP-based BCI system

\section{B. Using Amplitudes of Raw Signals (RS)}

In the first method, we used the amplitudes of the frequencies of the stimuli $\left(f_{1}=22 \mathrm{~Hz}\right.$ and $\left.f_{2}=27 \mathrm{~Hz}\right)$ as the feature vector for the classification. Let us denote the EEG signals recorded in the $n$-th trial as $\boldsymbol{X}_{n} \in \mathbb{R}^{30 \times 5120}$, while 30 is the number of EEG channels and 5120 is the temporal length of $10 \mathrm{~s}$ EEG signals. In this method, the feature vector $\boldsymbol{z}_{n}$ for $\boldsymbol{X}_{n}$ is obtained

$$
\boldsymbol{z}_{n}=\left[\begin{array}{c}
\operatorname{Amp}_{\left(f_{1}\right)}\left(\boldsymbol{X}_{n}\right) \\
\operatorname{Amp}_{\left(f_{2}\right)}\left(\boldsymbol{X}_{n}\right)
\end{array}\right],
$$

where $\operatorname{Amp}_{(f)}(\boldsymbol{X})$ is the function that returns the amplitude of FFT (Fast Fourier Transform) on the frequency $f$ from each channel of the signals $\boldsymbol{X}$. In our experiment, the signals from 30-channel EEG sernsors were recorded, and therefore each $\boldsymbol{z}_{n}$ has the form of $\mathbb{R}^{60 \times 1}$ vector. To build the classification model to discriminate between $\boldsymbol{z}_{n \in \mathrm{L}}$ and $\boldsymbol{z}_{n \in \mathrm{R}}$, we used a linear support vector machine (SVM). The performance of the classification will be presented in Section III.

\section{CSP for SSSEP Analysis}

To improve the classification performance, we applied CSP to the raw signals, then compared the amplitudes of extracted signal components, as did in [5]. From the recorded signals $\boldsymbol{X}$, we calculated two covariance matrices for each class as

$$
\begin{aligned}
& \boldsymbol{\Sigma}_{\mathrm{L}}=\frac{1}{N_{\mathrm{L}}} \sum_{n \in \mathrm{L}} \boldsymbol{X}_{n} \boldsymbol{X}_{n}^{\top}, \\
& \boldsymbol{\Sigma}_{\mathrm{R}}=\frac{1}{N_{\mathrm{R}}} \sum_{n \in \mathrm{R}} \boldsymbol{X}_{n} \boldsymbol{X}_{n}^{\top},
\end{aligned}
$$

where $N_{\mathrm{L}}$ and $N_{\mathrm{R}}$ are the number of trials in each class. From the sum of two matrices $\boldsymbol{\Sigma}=\boldsymbol{\Sigma}_{\mathrm{L}}+\boldsymbol{\Sigma}_{\mathrm{R}}$, we measured the whitening matrix $\boldsymbol{P}$, satisfying $\boldsymbol{P}^{\top} \boldsymbol{\Sigma} \boldsymbol{P}=\boldsymbol{I}$. If the eigendecomposition of $\boldsymbol{\Sigma}$ has the form $\boldsymbol{\Sigma}=\boldsymbol{U} \boldsymbol{\Lambda} \boldsymbol{U}^{\top}$, the whitening matrix $\boldsymbol{P}$ can be measured by $\boldsymbol{P}=\boldsymbol{U} \boldsymbol{\Lambda}^{-\frac{1}{2}}$.

Let us consider $\boldsymbol{V}$ that can diagonalize $\boldsymbol{P}^{\top} \boldsymbol{\Sigma}_{\mathrm{L}} \boldsymbol{P}$. Rewrite $\boldsymbol{P}^{\top} \boldsymbol{\Sigma} \boldsymbol{P}=\boldsymbol{I}$ to $\boldsymbol{P}^{\top}\left(\boldsymbol{\Sigma}_{\mathrm{L}}+\boldsymbol{\Sigma}_{\mathrm{R}}\right) \boldsymbol{P}=\boldsymbol{I}$, and apply $\boldsymbol{V}$ to both sides of the equation as

$$
\boldsymbol{V}^{\top}\left(\boldsymbol{P}^{\top} \boldsymbol{\Sigma}_{\mathrm{L}} \boldsymbol{P}+\boldsymbol{P}^{\top} \boldsymbol{\Sigma}_{\mathrm{R}} \boldsymbol{P}\right) \boldsymbol{V}=\boldsymbol{I}
$$

With considering that both of $\boldsymbol{V}^{\top}\left(\boldsymbol{P}^{\top} \boldsymbol{\Sigma}_{\mathrm{L}} \boldsymbol{P}\right) \boldsymbol{V}$ and $\boldsymbol{V}^{\top}\left(\boldsymbol{P}^{\top} \boldsymbol{\Sigma}_{\mathrm{R}} \boldsymbol{P}\right) \boldsymbol{V}$ are diagonal matrices, the spatial filter matrix $\boldsymbol{W}=\boldsymbol{P} \boldsymbol{V}$ can diagonalize $\boldsymbol{\Sigma}_{\mathrm{L}}$ and $\boldsymbol{\Sigma}_{\mathrm{R}}$ simultaneously. Meanwhile, if $i$-th diagonal element of $\boldsymbol{W}^{\top} \boldsymbol{\Sigma}_{\mathrm{L}} \boldsymbol{W}$ is $\lambda_{i}$, the $i$-th diagonal element of $\boldsymbol{W}^{\top} \boldsymbol{\Sigma}_{\mathrm{R}} \boldsymbol{W}$ should be $1-\lambda_{i}$. $\boldsymbol{W}$ can diagonalize both of $\boldsymbol{\Sigma}_{\mathrm{L}}$ and $\boldsymbol{\Sigma}_{\mathrm{R}}$, and corresponding diagonal values are reversely ordered. Therefore, a spatial filter $\boldsymbol{w}_{i}(i$-th column vector of $\boldsymbol{W}$ ) associated with larger $\lambda_{i}$ close to 1 , can maximize the variance of the projected signals of $\boldsymbol{X}_{n \in \mathrm{L}}$, while minimize the variance of the projected signals of $\boldsymbol{X}_{n \in \mathrm{R}}$.

To extract better spatial filters, which work more efficiently for each frequency band, we applied CSP method to already band-pass filtered signals for two frequency bands, respectively. We first obtained band-pass filtered signals $\boldsymbol{X}_{n\left(f_{1}\right)}$ and $\boldsymbol{X}_{n\left(f_{2}\right)}$ from the raw signals $\boldsymbol{X}_{n} . \boldsymbol{X}_{n\left(f_{1}\right)}$ is band-pass filtered 


\begin{tabular}{|c|c|}
\hline Amplitude increase in $22 \mathrm{~Hz}$ for attention to the left hand & Amplitude increase in $27 \mathrm{~Hz}$ for attention to the right hand \\
\hline$t_{c}=\frac{\mu_{n \in \mathrm{L}}-\mu_{n \in \mathrm{R}}}{\sqrt{\sigma_{n \in \mathrm{L}}^{2} / N_{L}+\sigma_{n \in \mathrm{R}}^{2} / N_{R}}}$ & $t_{c}=\frac{\mu_{n \in \mathrm{R}}-\mu_{n \in \mathrm{L}}}{\sqrt{\sigma_{n \in \mathrm{L}}^{2} / N_{L}+\sigma_{n \in \mathrm{R}}^{2} / N_{R}}}$ \\
\hline & \\
\hline Subject 2 & Subject 2 \\
\hline
\end{tabular}

Fig. 2. Amplitude differences

between $f_{1}-1 \mathrm{~Hz}$ to $f_{1}+1 \mathrm{~Hz}$, and $\boldsymbol{X}_{n\left(f_{2}\right)}$ is filtered between $f_{2}-1 \mathrm{~Hz}$ to $f_{2}+1 \mathrm{~Hz}$,

We calculated the covariance matrices $\boldsymbol{\Sigma}_{\mathrm{L}\left(f_{1}\right)}$ and $\boldsymbol{\Sigma}_{\mathrm{R}\left(f_{1}\right)}$ from $\boldsymbol{X}_{(n \in \mathrm{L})\left(f_{1}\right)}$ and $\boldsymbol{X}_{(n \in \mathrm{R})\left(f_{1}\right)}$, and calculated $\boldsymbol{\Sigma}_{\mathrm{L}\left(f_{2}\right)}$ and $\boldsymbol{\Sigma}_{\mathrm{R}\left(f_{2}\right)}$ from $\boldsymbol{X}_{(n \in \mathrm{L})\left(f_{2}\right)}$ and $\boldsymbol{X}_{(n \in \mathrm{R})\left(f_{2}\right)}$, as we did in (2) and (3). By the CSP approach, we obtained the spatial filter matrices $\boldsymbol{Q}$ and $\boldsymbol{R}$. $\boldsymbol{Q}$ can diagonalize $\boldsymbol{\Sigma}_{\mathrm{L}\left(f_{1}\right)}$ and $\boldsymbol{\Sigma}_{\mathrm{R}\left(f_{1}\right)}$ simultaneously, while $\boldsymbol{R}$ can diagonalize $\boldsymbol{\Sigma}_{\mathrm{L}\left(f_{2}\right)}$ and $\boldsymbol{\Sigma}_{\mathrm{R}\left(f_{2}\right)}$.

From $\boldsymbol{Q}$ and $\boldsymbol{R}$, we collected the eigenvectors corresponding to the four largest and four smallest eigenvalues and denoted them by

$$
\begin{aligned}
\tilde{\boldsymbol{Q}} & =\left[\boldsymbol{q}_{1}, \cdots, \boldsymbol{q}_{4}, \boldsymbol{q}_{27}, \cdots, \boldsymbol{q}_{30}\right], \\
\tilde{\boldsymbol{R}} & =\left[\boldsymbol{r}_{1}, \cdots, \boldsymbol{r}_{4}, \boldsymbol{r}_{27}, \cdots, \boldsymbol{r}_{30}\right]
\end{aligned}
$$

$\tilde{\boldsymbol{Q}}$ is the discriminative filter for $\boldsymbol{X}_{n\left(f_{1}\right)}$, while $\tilde{\boldsymbol{R}}$ is the filter for $\boldsymbol{X}_{n\left(f_{2}\right)}$. Therefore, we measured the amplitude of $\tilde{\boldsymbol{Q}}^{\top} \boldsymbol{X}_{n\left(f_{1}\right)}$ on $f_{1} \mathrm{~Hz}$, and the amplitude of $\tilde{\boldsymbol{R}}^{\top} \boldsymbol{X}_{n\left(f_{2}\right)}$ on $f_{2}$ $\mathrm{Hz}$. By concatenating the amplitudes, we obtained the feature vector $\boldsymbol{z}_{n}^{\prime} \in \mathbb{R}^{16 \times 1}$ corresponding to $\boldsymbol{X}_{n}$ :

$$
\boldsymbol{z}_{n}^{\prime}=\left[\begin{array}{c}
\operatorname{Amp}_{\left(f_{1}\right)}\left(\tilde{\boldsymbol{Q}}^{\top} \boldsymbol{X}_{n\left(f_{1}\right)}\right) \\
\operatorname{Amp}_{\left(f_{2}\right)}\left(\tilde{\boldsymbol{R}}^{\top} \boldsymbol{X}_{n\left(f_{2}\right)}\right)
\end{array}\right]
$$

Then, the classification model for discriminating $\boldsymbol{z}_{n \in \mathrm{L}}^{\prime}$ and $\boldsymbol{z}_{n \in \mathrm{R}}^{\prime}$ was trained.

\section{EXPERIMENTS}

\section{A. Spatial Patterns of SSSEP}

In [5], we showed that CSP can extract useful spatial filters emphasizing the difference between the signals in $\boldsymbol{L}$ and $\boldsymbol{R}$. However, they did not extensively considered about which channels (or brain region) are crucial for the SSSEP analysis due to their different responses to each attention. To prove the importance of the spatial information for SSSEP analysis, and support the idea of applying CSP, we examined the spatial pattern difference between $\boldsymbol{L}$ and $\boldsymbol{R}$.

To evaluate the differences in a statistical manner, we measured $t$-values from Welch's $t$-test for channel $c$, such that

$$
t_{c}=\frac{\mu_{n \in \mathrm{L}}-\mu_{n \in \mathrm{R}}}{\sqrt{\frac{\sigma_{n \in \mathrm{L}}^{2}}{N_{\mathrm{L}}}+\frac{\sigma_{n \in \mathrm{R}}^{2}}{N_{R}}}}
$$

where $\mu_{n \in \mathrm{L}}$ and $\sigma_{n \in \mathrm{L}}$ are the mean value and standard deviation of $\boldsymbol{z}_{n \in \mathrm{L}}$, while $\mu_{n \in \mathrm{R}}$ and $\sigma_{n \in \mathrm{R}}$ are the values for $\boldsymbol{z}_{n \in \mathrm{R}}$. This $t$ value is proportional to the difference between two groups, but inversely proportional to the standard deviations of each group. Therefore, $t$ provides better measurement to evaluate the discriminability than $\mu_{n \in \mathrm{L}}-\mu_{n \in \mathrm{R}}$.

To measure the amplitude change of $\boldsymbol{L}$ against $\boldsymbol{R}$ on $f_{1}$, we measured $t$ values and illustrated them by a topographic map in the left column of Fig. 2. We can see that the amplitude decreases were mainly occurred on the right hemisphere of the brain. We also denoted the channel with the highest discriminability having the maximum $|t|$ value by " $x$ " marks in the plots. For all subjects, the " $\times$ " marks were located in the right side of the brain. To clarify the meaning of the values in Fig. 2, we plotted each trial's amplitudes in the channels with the maximum discriminability in Fig. 3 with error bars represent the mean and standard deviation for each class.

The amplitude change of $\boldsymbol{R}$ against $\boldsymbol{L}$ on $f_{2}$ was also illustrated in the right column of Fig. 2. For the illustration, we modified (8) with replacing $\mu_{n \in \mathrm{L}}-\mu_{n \in \mathrm{R}}$ to $\mu_{n \in \mathrm{R}}-\mu_{n \in \mathrm{L}}$. In contrast to the results in the left column, the amplitude decreases were mainly observable on the left hemisphere, and the channels with the highest discriminability were located in the left side of the brain. Before the experiments, we assumed that the attention on the one hand will increase the amplitudes on the channels in the contralateral side, because of the neurological background that the somatosensory cortices for each hand is located in the contralateral sides of the hands. However, experimental results showed that the attention decreases the amplitudes, rather than increases. Although this decrease is not in agreement with the background, we identified that the amplitude change from the attention is mainly observable in the contralateral side of the attended hand.

We also assumed that the amplitude changes will be dominantly observed in the parietal region near the somatosensory cortex. However, the experimental results showed that the frontal region is also sensitive to the change of attention.

\section{B. Discriminative Spatial Patterns from CSP}

In the next step, we investigated the spatial pattern from the CSP method. From the spatial filter matrix $\boldsymbol{W}$, the spatial pattern vector $\boldsymbol{a}_{i}$ corresponding to $i$-th signal component $\boldsymbol{w}_{i}^{\top} \boldsymbol{X}$ is obtained form $i$-th row vector of $\boldsymbol{W}^{-1}$. The $c$-th value 


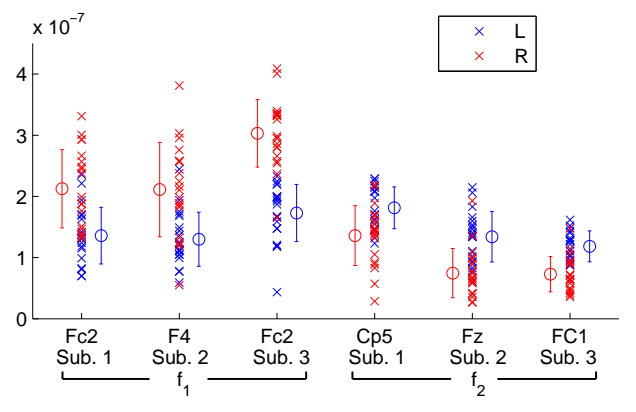

Fig. 3. Amplitudes in the channels with the maximum discriminability

TABLE I

ClassificATION ACCURACY

\begin{tabular}{c|c|c|c|c}
\hline \multirow{2}{*}{ Subjects } & \multicolumn{2}{|c|}{ RS } & \multicolumn{2}{c}{ CSP } \\
\cline { 2 - 5 } & Session 1 & Session 2 & Session 1 & Session 2 \\
\hline 1 & 67.5 & 60.0 & 57.5 & 85.0 \\
\hline 2 & 87.5 & 87.5 & 70.0 & 85.0 \\
\hline 3 & 85.0 & 97.5 & 90.0 & 95.0 \\
\hline
\end{tabular}

in vector $\boldsymbol{a}_{i}$ signifies the influence of $i$-th signal component to channel $c$ of the original signals $\boldsymbol{X}$.

We plotted the obtained spatial pattern vectors in Fig. 4. We used sixteen spatial filters (eight for $\tilde{\boldsymbol{Q}}$ and eight for $\tilde{\boldsymbol{R}}$ ), so there are sixteen spatial pattern vectors corresponding to each spatial filter. Among these vectors, we only plotted the vector with the maximum discriminability, showing the largest difference between the feature values for $\boldsymbol{L}$ and $\boldsymbol{R}$. To evaluate the difference, we used Welch's $t$-test again. We measured the feature vectors $\boldsymbol{z}_{n}^{\prime}$ for each trial, and collected the vectors for each class as $\boldsymbol{Z}_{n \in \mathrm{L}} \in \mathbb{R}^{16 \times 20}$ and $\boldsymbol{Z}_{n \in \mathrm{L}} \in \mathbb{R}^{16 \times 20}$ $\left(N_{\mathrm{L}}=N_{\mathrm{R}}=20\right)$. From each row of $\boldsymbol{Z}_{n \in \mathrm{L}}$ and $\boldsymbol{Z}_{n \in \mathrm{R}}$, we calculated the mean and variance, then measured $t$ value by (8). We selected the spatial filters with the highest and lowest $t$ value, then plotted their corresponding spatial pattern vectors in Fig. 4 with the distributions of obtained feature values $\boldsymbol{z}_{n}^{\prime}$.

We could see that the pattern vectors corresponding to the highest $t$ value (in the left column) emphasize the right side of the posterior region, while the vectors corresponding to the lowest $t$ value (in the right column) emphasize the left side of the same region. As we mentioned in the previous subsection, this pattern is in agreement with the neurological background about somatosensory cortex. In Fig. 4, the symmetry between the results in each column was clearer than the results in Fig. 2 , and the separability between the feature points for each class was also enhanced than the results in Fig. 3.

We also denoted the classification accuracy from fivefold cross-validation test in Table I, to identify the performance improvement from the CSP approach. Although, CSP made clearly separable clusters for each class in Fig. 4, it could not achieve clear performance improvement in the test.

\section{CONCLUSION}

In this study, we examined the difference of the spatial patterns between SSSEP for two different attentions: attention

\begin{tabular}{|c|c|c|}
\hline Sub. & $\begin{array}{l}\text { Pattern with the } \\
\text { highest } t \text { value }\end{array}$ & $\begin{array}{l}\text { Pattern with the } \\
\text { lowest } t \text { value }\end{array}$ \\
\hline 1 & & \\
\hline 2 & 60 & \\
\hline 3 & & \\
\hline & & \\
\hline
\end{tabular}

Fig. 4. Spatial patterns from CSP

to the tactile stimulus on 1) the left hand, and 2) the right hand. Experimental results showed that the attention to one hand decreases the amplitudes of the given frequency in the contralateral side, especially in the posterior region. Next experiment showed that CSP can emphasize this spatial pattern change with achieving better separability in the feature space for the classification. We believe this observation will be beneficial for evaluating the adaptability of users to SSSEPbased BCI, or designing novel algorithms or methods.

\section{ACKNOWLEDGMENTS}

This work was supported by National Research Foundation (NRF) of Korea (NRF-2010-0018829 and NRF2013R1A2A2A01067464) and POSTECH Rising Star Program.

\section{REFERENCES}

[1] J. R. Wolpaw, N. Birbaumer, D. J. McFarland, G. Pfurtscheller, and T. M. Vaughan, "Brain-computer interfaces for communication and control," Clinical Neurophysiology, vol. 113, pp. 767-791, 2002.

[2] G. R. Müller-Putz, R. Scherer, and G. Pfurtscheller, "Steady-state somatosensory evoked potentials: Suitable brain signals for brain computer interfaces?" IEEE Transactions on Neural Systems and Rehabilitation Engineering, vol. 14, pp. 30-37, 2006.

[3] D. Zhang, Y. Wang, A. Maye, A. K. Engel, X. Gao, B. Hong, and S. Gao, "A brain-computer interface based on multi-modal attention," in Proceedings of IEEE EMBS Conference on Neural Engineering, Kohala Coast, Hawaii, 2007, pp. 414-417.

[4] S. Ahn and S. C. Jun, "Feasibility of hybrid BCI using ERD- and SSSEPBCI," in International Conference on Control, Automation and Systems, Jeju Island, Korea, 2012, pp. 2053-2056.

[5] Y. Nam, A. Cichocki, and S. Choi, "Common spatial patterns for steadystate somatosensory evoked potentials," in Proceedings of IEEE EMBS Conference, Osaka, Japan, 2013, pp. 2255-2258.

[6] Z. J. Koles, "The quantitative extraction and topographic mapping of the abnormal components," EEG and Clinical Neurophysilology, vol. 79, pp. 440-447, 1991.

[7] H. Ramoser, J. Müller-Gerking, and G. Pfurtscheller, "Optimal spatial filtering of single trial EEG during imagined hand movement," IEEE Transactions on Rehabilitation Engineering, vol. 8, pp. 441-446, 2000. 